\title{
MANIFESTACIONES OFTALMOLÓGICAS EN LA ENFERMEDAD DE FABRY. A PROPÓSITO DE 4 CASOS CON ACTIVIDAD DEFICIENTE DE a-GALACTOSIDASA A
}

\section{OPHTHALMOLOGICAL MANIFESTATIONS IN FABRY'S DISEASE. FOUR CLINICAL CASES SHOWING DEFICIENT ALPHA-GALACTOSIDASE-A ACTIVITY}

\author{
RODRÍGUEZ-GONZÁLEZ-HERRERO ME ${ }^{1}$, MARÍN-SÁNCHEZ JM ${ }^{2}$, GIMENO JR ${ }^{3}$, \\ MOLERO-IZQUIERDO C ${ }^{4}$, DE-CASAS-FERNÁNDEZ A ${ }^{4}$, RODRÍGUEZ-GONZÁLEZ-HERRERO B ${ }^{4}$, \\ SAN-ROMÁN I ${ }^{4}$, LOZANO J ${ }^{4}$, DE-LA-MORENA G ${ }^{3}$, LLOVET-OSUNA F ${ }^{2}$
}

\section{RESUMEN}

Caso clínico: La enfermedad de Fabry es una enfermedad producida por una alteración en el catabolismo de los glucoesfingolípidos. Se muestran las alteraciones oftalmológicas de cuatro pacientes detectados tras evaluar desde el punto de vista analítico, cardiológico y genético a 113 enfermos.

Discusión: La enfermedad de Fabry es una enfermedad infrecuente con afectación oftalmológica inconstante existiendo enfermos con Fabry sin afectación ocular y portadores sanos, con importantes alteraciones oculares. El depósito de glucoesfingolípidos produce afectación a nivel corneal, cristaliniano, vascular y retiniano. Las alteraciones vasculares afectan no sólo a las venas sino también a las arterias como mostramos en nuestros pacientes.

Palabras clave: Enfermedad de Fabry, alfa-galactosidasa A, vasculopatía.

\begin{abstract}
Case report: Fabry's disease is an illness produced by an alteration in the catabolism of the glycosphingolipids. We report ophthalmologic findings in 4 people, detected after 113 patient evaluations from an analytical, cardiological and genetic point of view. Discussion: Fabry's disease is uncommon and shows variable ophthalmologic affectation. Some patients with Fabry's disease do not present ocular affectation, while, on the other hand, healthy carriers with important ocular alterations have been described. The deposit of glycosphingolipids produces affectation at the corneal, crystalline, vascular and retinal levels. The vascular alterations affect not only the veins but also the arteries, as we report in our patients (Arch Soc Esp Oftalmol 2008; 83: 713-718).
\end{abstract}

Key words: Fabry's Disease, alpha-galactosidaseA, vasculopathy.

\footnotetext{
Recibido: 28/5/07. Aceptado: 21/11/08.

Hospital Universitario Virgen de la Arrixaca. Universidad de Murcia. Murcia. España.

${ }^{1}$ Doctor en Medicina. Profesor Asociado Universidad. Especialista en Oftalmología.

2 Licenciado en Medicina. Especialista en Oftalmología.

3 Doctor en Medicina. Especialista en Cardiología.

${ }^{4}$ Licenciado en Medicina.

Comunicación presentada en el LXXXII Congreso de la S.E.O. (A Coruña 2006).

Correspondencia:

M. ${ }^{a}$ Elena Rodríguez González-Herrero

Edificio Avenida

Avda. Europa, 26, 1. ${ }^{\text {a }}$ Esc. 3. ${ }^{\circ}$ B

30007 Murcia

España

E-mail: mariaelenargh@ @otmail.com
} 


\section{INTRODUCCIÓN}

La enfermedad de Fabry es una rara enfermedad sistémica, recesiva ligada a $X$, producida por una alteración en el catabolismo de los glucoesfingolípidos, debido a una actividad deficiente de la enzima lisosomal a-galactosidasa A (AaGA; fig. 1). Con una prevalencia de 1/117000 personas, produce alteraciones en distintos órganos, principalmente a nivel neurológico, cardiológico, dermatológico, renal y oftalmológico (1).

Se presentan las manifestaciones oftalmológicas de cuatro pacientes (tres afectos de enfermedad de Fabry y un portador) que fueron detectados tras el estudio cardiológico de 113 pacientes afectos de Miocardiopatía Hipertrófica (MCH) por el Servicio de Cardiología del Hospital Virgen de la Arrixaca. Para el diagnóstico se consideró patológicos unos niveles de AaGA $<30 \%$ en varones y $<50 \%$ en mujeres y se realizó un estudio genético de la región cromosómica Xq22 en cada paciente.

\section{CASOS CLÍNICOS}

El Servicio de Oftalmología del Hospital Universitario Virgen de la Arrixaca, ha explorado a cuatro pacientes (tres pacientes afectos de enfermedad de Fabry y un portador). El paciente uno (p1) presentó una delección en el exón 7 (1072-74 delGAG) y tres pacientes $(\mathrm{p} 2, \mathrm{p} 3, \mathrm{p} 4)$ presentaron una alteración en el exón 6 (Asp313Tyr).

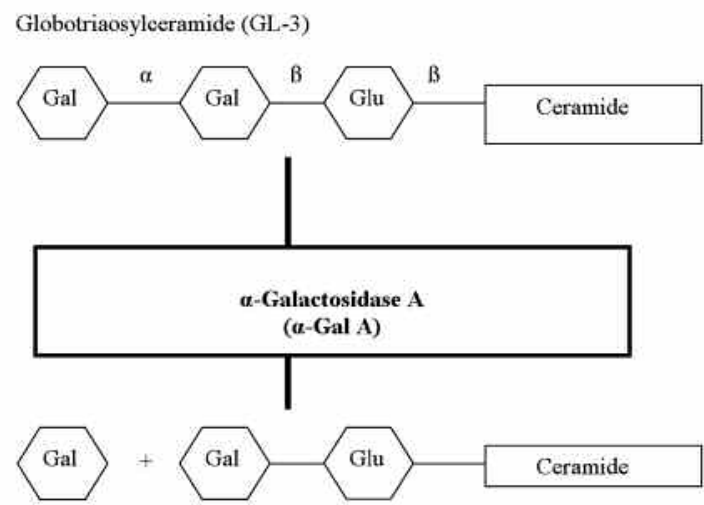

Fig. 1: Se muestra un esquema del catabolismo de la Globotriosilceramida (GL3) a Lactosilceramida mediante la enzima a-Galactosidasa A.

\section{Caso 1}

Varón de 52 años afecto de Enfermedad de Fabry que forma parte de la familia H171 (gráfica 1). Presenta una agudeza visual corregida (AVC) 0,9 en ambos ojos (AOs), pingüeculas nasales bilaterales, dilatación de los vasos conjuntivales (fig. 2), córnea verticilata (fig. 3), movilización pigmentaria a nivel del polo posterior con incremento de la pigmentación superior a la papila y aumento de la tortuosidad vascular arterial y venosa que se confirma por AFG (fig. 4). Resto exploración oftalmológica normal.

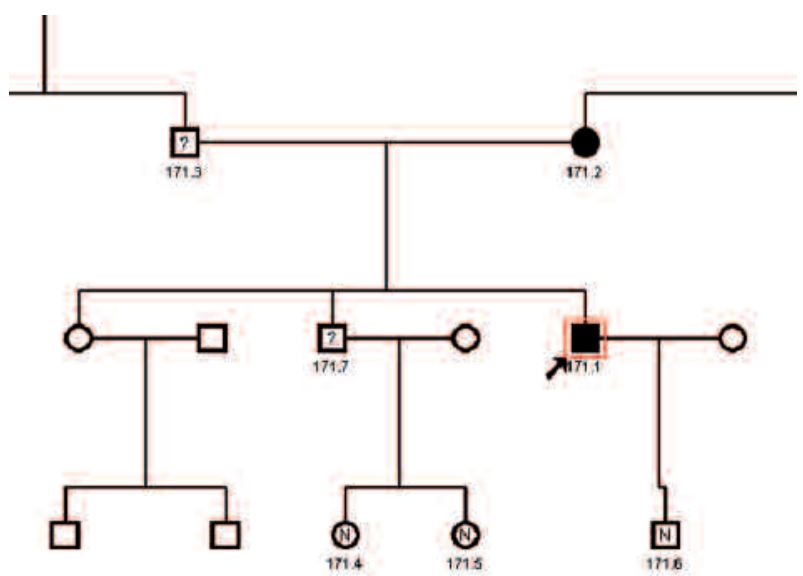

Diagrama 1. Se muestra el árbol genealógico de la familia H171.

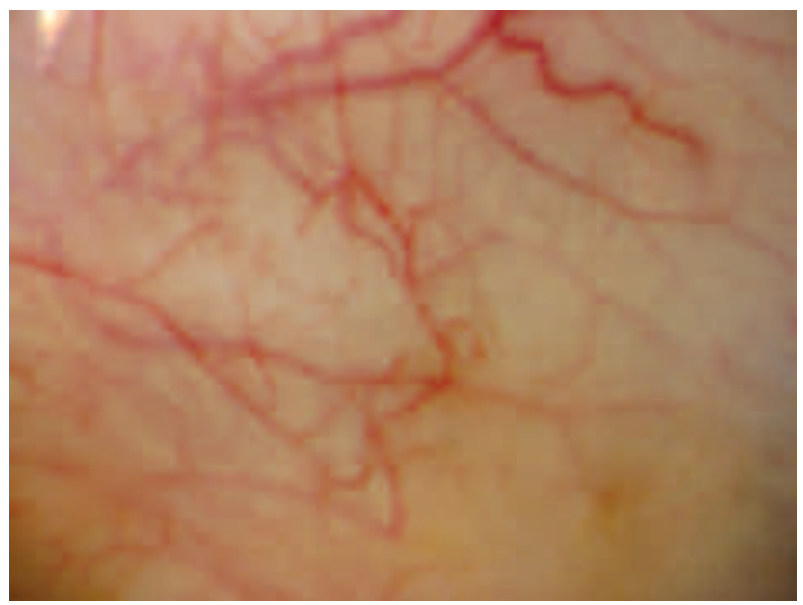

Fig. 2: Alteraciones vasculares conjuntivales, con irregularidad del calibre y ectasias vasculares del paciente uno. 


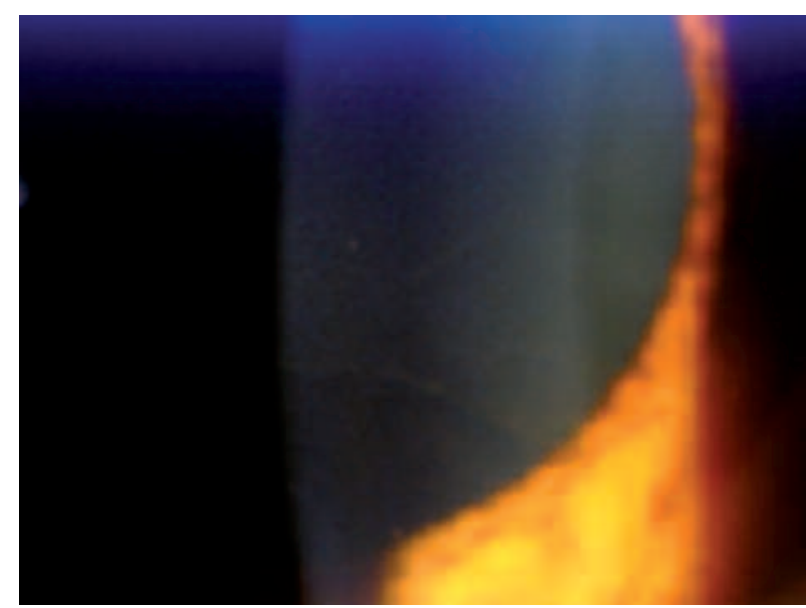

Fig. 3: Depósitos corneales epiteliales de ceramidas del paciente uno.

\section{Caso 2}

Varón de 81 años diagnosticado de EPOC, enfermedad de Fabry y MCH. Requiere marcapasos. Forma parte de la familia H202. La AVC es 0,3 OD. Amaurosis OI secundaria a traumatismo. No se detectan signos de Fabry.

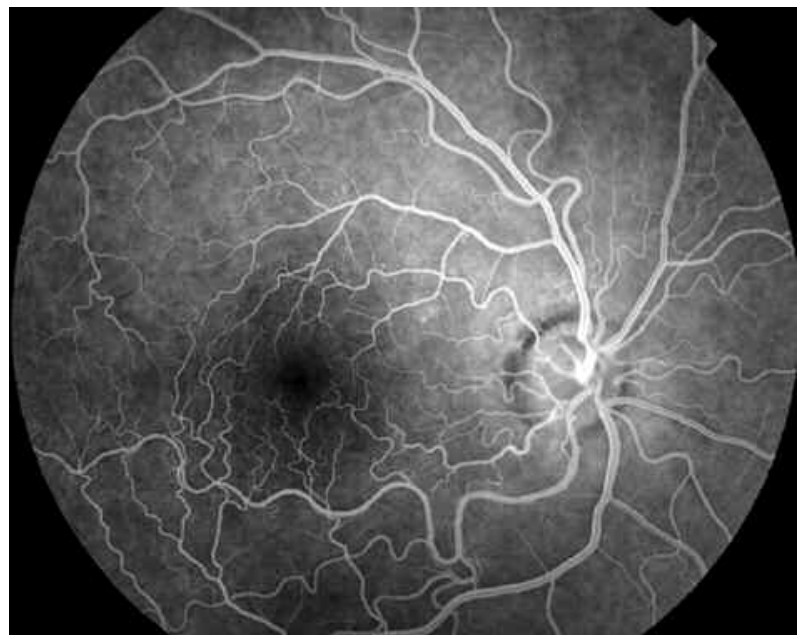

Fig. 4: Angiografía (AFG) en la que se muestra el incremento en la tortuosidad arterial y venosa a nivel del polo posterior del OD del paciente uno.

\section{Caso 3}

Varón de 45 años, sano, portador de la mutación de la alfa-galactosidasa. Forma parte de la familia H90 (gráfica 2). La exploración oftalmológica muestra: AVC 0,8 OD y 0,9 OI, dilatación de los vasos conjuntivales (fig. 5), córnea deslustrada
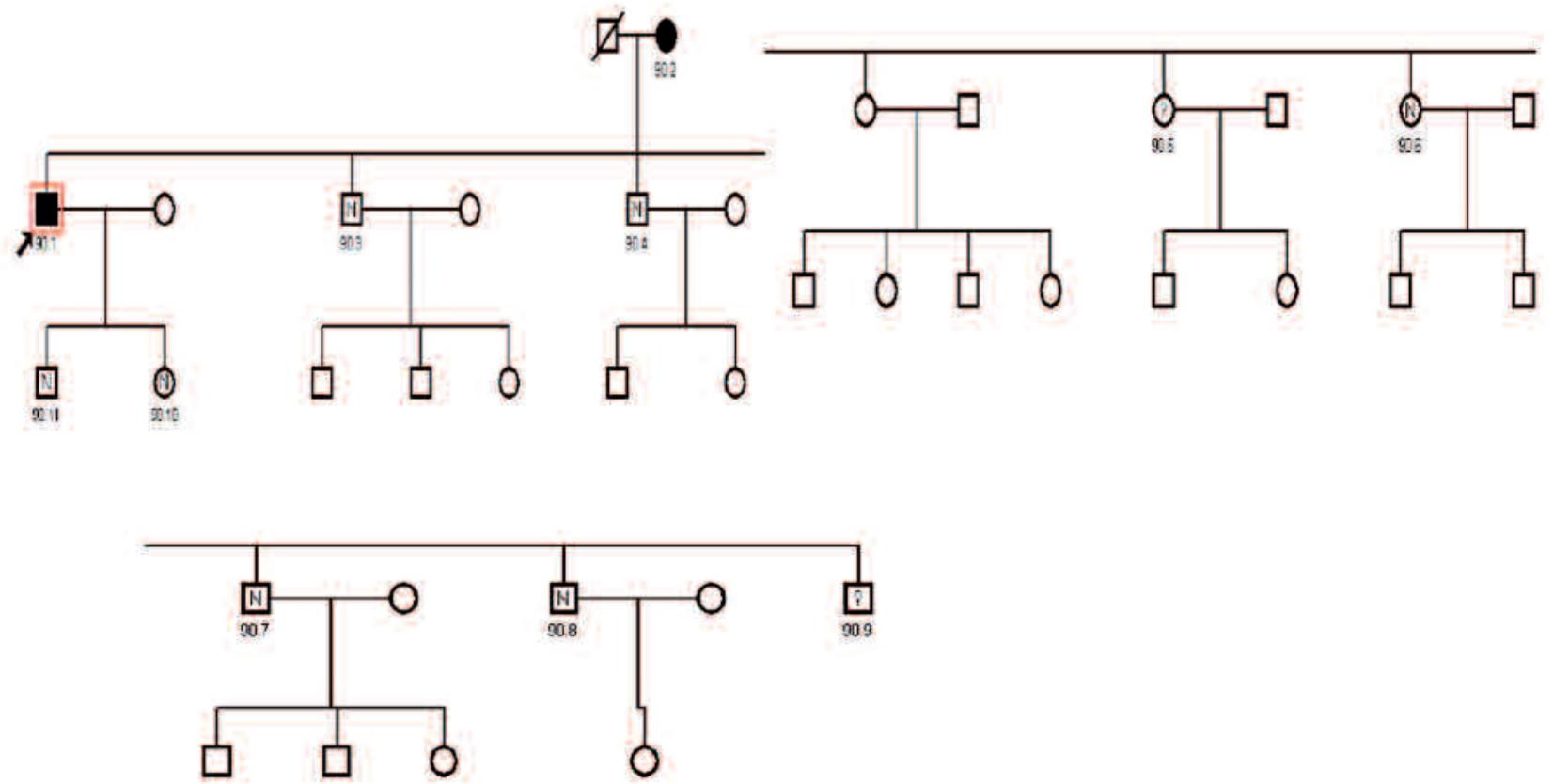

Diagrama 2. Se muestra el árbol genealógico de la familia H90. 


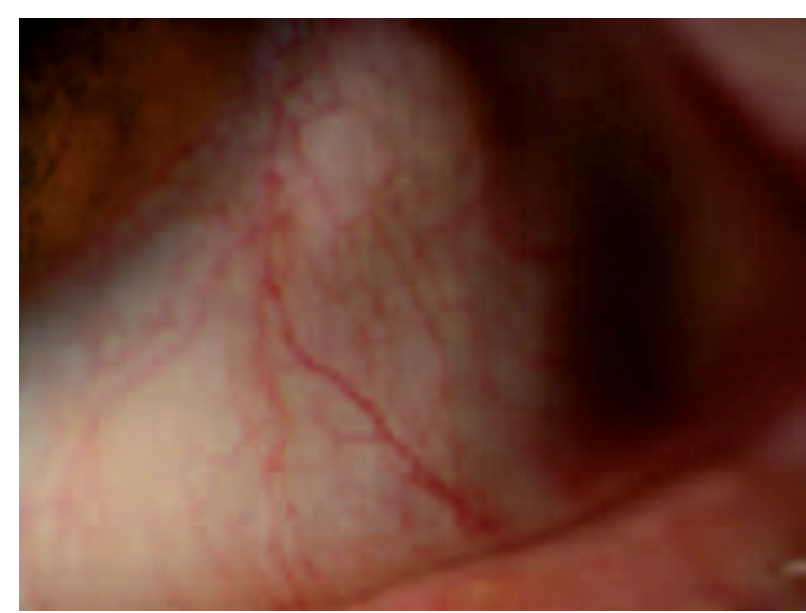

Fig. 5: Alteraciones vasculares conjuntivales del paciente tres. con disminución de la transparencia, y opacidades puntiformes cristalinianas ecuatoriales, movilización pigmentaria a nivel del polo posterior y ecuatorial con alteraciones del epitelio pigmentario retiniano.

\section{Caso 4}

Varón de 56 años, enfermo de Fabry y afecto de $\mathrm{MCH}$. Forma parte de la familia H90 (gráfica 2). La exploración oftalmológica revela: AVC: 1 AOs, leve facoesclerosis bilateral, ectasias de los vasos conjuntivales, cornea verticilada y alteraciones pigmentarias a nivel de polo posterior en fondo de ojo y AFG (fig. 6).

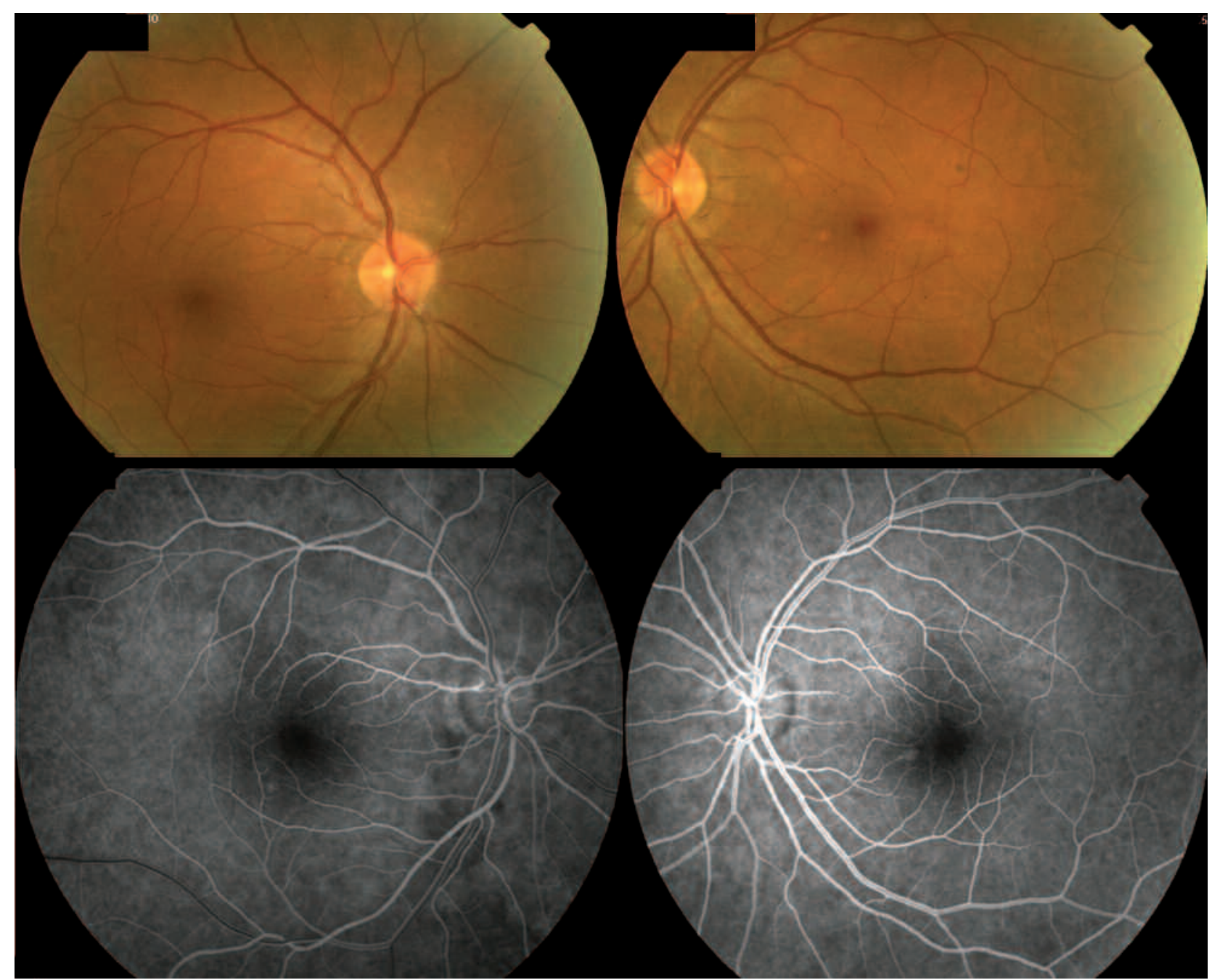

Fig. 6: Retinografías y AFG del paciente 4 que muestran las alteraciones del epitelio pigmentario a nivel macular en ambos ojos. Destaca la normalidad en el calibre y regularidad vascular. 


\section{DISCUSIÓN}

La enfermedad de Fabry es una enfermedad infrecuente que produce múltiples patologías a nivel sistémico y oftalmológico. Los pacientes descritos presentan alteraciones cardiológicas indistinguibles de los pacientes afectos de Miocardiopatía Hipertrófica con AaGA normal, por lo que la existencia de alteraciones oftalmológicas compatibles con enfermedad de Fabry apoya el diagnóstico. La afectación oftalmológica es inconstante pudiendo existir enfermos de Fabry sin enfermedad ocular (paciente n. ${ }^{\circ}$ 2) y pacientes sanos portadores de Fabry con manifestaciones oftalmológicas características (paciente n. $^{\circ}$ 3).

Como patología oftalmológica se han descrito: alteraciones vasculares, edema periorbitario, córnea verticilata, cataratas, pigmentación retiniana periférica, papiledema, oclusión de la arteria central de la retina, atrofia óptica, discromatopsia, nistagmus y oftalmoplejía internuclear $(1-2,4)$.

La afección corneal es la más frecuente. Lo más característico es el depósito de glucoesfingolípidos entre la membrana basal del epitelio corneal y la membrana de Bowman produciendo el patrón de afectación corneal en "córnea verticilada», que también se observa en pacientes afectos de enfermedad de Tangier, Melanoqueratosis estriada, Síndrome de Melkersson-Rosenthal y secundarios a fármacos como Amiodarona o Cloroquina. En el paciente n. $^{\circ} 1$, puesto que estaba en tratamiento crónico con Amiodarona, se realizó control oftalmológico 6 meses después de discontinuar este tratamiento, apreciando córnea verticilada en el mismo estado que el referido, lo que sugiere que los depósitos podrían ser secundarios a Fabry.

La afección cristaliniana característica consiste en la opacificación de las suturas cristalinianas a nivel posterior produciendo la catarata de Fabry. Existen otras alteraciones cristalinianas como facoesclerosis, depósitos y opacidades cristalinianas inespecíficas.

Las lesiones conjuntivales y retinianas vasculares forman parte de la afectación sistémica de pequeño vaso por Fabry $(3,5)$ y es secundaria a depósitos intracitoplasmáticos en el endotelio vascular. Las dilataciones aneurismáticas, angulaciones y segmentación de los vasos conjuntivales se producen en el $60 \%$ de los enfermos de Fabry pero no se han referenciado en enfermos portadores de la enfermedad ni afectando a las arterias retinianas como en nuestros pacientes.

La enfermedad de Fabry es una enfermedad infrecuente sin embargo la detección de la afectación oftalmológica por Fabry facilita el diagnóstico precoz de la misma y permite un tratamiento temprano evitando las lesiones irreversibles producidas por los depósitos de GL-3.

\section{BIBLIOGRAFÍA}

1. Iester M, Sodi A, Ocella C, Vittone P. Ocular finding in Fabry's disease. Contrib Nephrol 2001; 136: 260-262.

2. Orssaud C, Dufier J, Germain D. Ocular manifestations in fabry disease: a survey of 32 hemizygous male patients. Ophthalmic Genet 2003; 24: 129-139.

3. Dichgans $M$. A new cause of hereditary small vessel disease. Angiopathy of retina and brain. Neurology 2003; 60: 8-9.

4. Nguyen TT, Gin T, Nicholls K, Low M, Galanos J, Craford A. Ophthalmological manifestations of fabry disease: a survey of patiens at the Royal Melbourne Fabry disease treatment centre. Clin Exp Ophthalmol 2005; 33: 164168.

5. Sodi A, Ioannidis A, Mehta A, Davey C, Beck M, Pitz S. Ocular manifestations of Fabry disease: data from the Fabry Outcome Survey. Br J Ophthalmol 2007; 91: 210214. 